\title{
FACTORS INFLUENCING THE ANTIPLATELET THERAPY IN PATIENTS REQUIRING VASCULAR SURGERY.
}

Sellitto Carmine $\mathrm{MD}^{1}$, Conti Valeria $\mathrm{PhD}^{1,2}$, Manzo Valentina $\mathrm{PhD}^{1}$, lannaccone Teresa $\mathrm{MD}^{1}$, Costantino Maria PhD ${ }^{1,3}$, Torsiello Martina PharD ${ }^{1}$, Di Fiore Giovanni PharD ${ }^{1}$, Accarino Giancarlo $\mathrm{MD}^{4}$, Nicolella Giovanna MD , Corbi Graziamaria $\mathrm{PhD}^{5}$, Filippelli Amelia $\mathrm{PhD}^{1,2}$

${ }^{1}$ Department of Medicine, Surgery and Dentistry "Scuola Medica Salernitana", University of Salerno- S. Allende street, 84081-Baronissi (Salerno), +39 089965226. Italy.

${ }^{2}$ Clinical Pharmacology and Pharmacogenetics Unit, University Hospital "San Giovanni di Dio e Ruggi d'Aragona", via S. Leonardo 1, Salerno- +39 089672424. Italy.

${ }^{3}$ Association non-profit F.I.R.S.Thermae (Interdisciplinary Training, Researches and spa Sciences) in Italian National Register of Research of MIUR, Via Marziale, 21, 80070 Bacoli, Naples, +39 377232 0882. Italy.

"Vascular surgery Unit, University Hospital “San Giovanni di Dio e Ruggi D’Aragona”, Salerno- via S. Leonardo 1, Salerno- +39089 673232. Italy

${ }^{5}$ Department of Medicine and Health Sciences, University of Molise, Via Francesco De Sanctis, 1, 86100 Campobasso- +39 08744041. Italy

\section{Introduction}

The antiplatelet agent clopidogrel (CLO) is a prodrug mainly activated by the CYP2C19. The CYP2C19-*2 and -*3 SNPs, called Loss of Function alleles, are associated to a phenotype of intermediate (IM) and poor metabolizers (PM). The CYP2C19- *17 SNP identifies the rapid and ultra-rapid metabolizers (UMs) (1). IMs and especially PMs might be not able to efficiently activate the drug and they are at greater risk of thrombotic events. ABCB1C3435T influences the impaired function of P-glycoprotein thereby hindering the CLO absorption. Patients with TT genotype have a higher rate of cardiovascular events than those with CC genotype. ABCB1C3435T SNP seems to influence ADP dependent platelet reactivity and T-allele carriers are likely to have a poor response to antiplatelet therapy (AT) (2). Proton Pump Inhibitors (PPIs), primarily omeprazole and esomeprazole, are inhibitors of CYP2C19. Actually, it has been shown that drug-drug interaction between PPIs and CLO might lead to an attenuated antiplatelet effect (3). Female gender has been associated with variable responses to AT enhancing basal platelet reactivity, resulting in high residual on-treatment platelet reactivity. In primary cardiovascular prevention, intake of aspirin would lead to a decrease in important coronary events in men, but not in women. Main aim of this study was to investigate the association between several factors influencing AT and platelet aggregation values in patients eligible for vascular surgery (VS) treated with AT.

\section{Methods}

This observational study is ongoing at University Hospital of Salerno: adult patients eligible for VS were enrolled at VS Unit. Demographic and clinical data of each patient were recorded in a Case Report Form. Once informed consent was obtained, blood samples were collected at pre-hospitalization visit (TO) and outpatient visit 1 month post-intervention (T1) to measure platelet reactivity by impedance aggregometry with ADPtest or ASPItest (fig. 1). In accordance to ADPtest, values $<470$ $\mathrm{AU}^{*} \min$ indicate a platelet function responsiveness, while values $<500 \mathrm{AU} * \min$ indicate a responsiveness for ASPItest. Genomic DNA was isolated to perform CYP2C19 and ABCB1 testing by Real Time PCR and pyrosequencing at Clinical Pharmacology and Pharmacogenetics Unit.

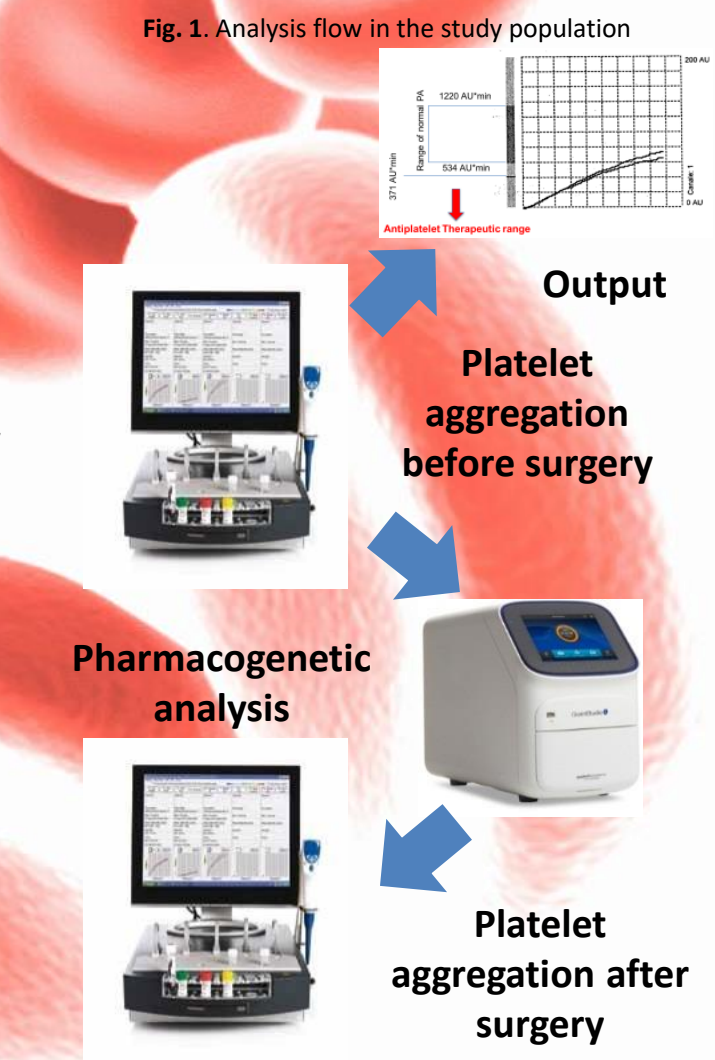




\section{FACTORS INFLUENCING THE ANTIPLATELET THERAPY IN PATIENTS REQUIRING VASCULAR SURGERY.}

\section{Results}

A total of 76 patients (63 male/13 female, average age: 70.6 years) were enrolled. Among them, 30 patients were on treatment with CLO, 53 with salicylates, 2 with ticlopidine, only one was treated with prasugrel and one with ticagrelor. 16 patients (10 male and 6 female) treated with CLO showed a residual platelet aggregation (RPA) (ADPtest $>470$ AU*min); 15/16 were also treated with PPIs. Almost all the patient who showed RPA were carriers of the aforementioned SNPs as follows: 5 wt for CYP2C19 but ABCB13435CT, 3 CYP2C19*1/*2 and ABCB13435CT, 2 CYP2C19*1/*2, 2 wt for CYP2C19 but ABCB13435TT. 3 were CYP2C19*1/*17 (one was also $A B C B 13435 C T$ and another also $A B C B 13435 T T$ ). Only one subject was wt for both CYP2C19 and ABCB1. 8 patients treated with salicylates showed RPA (>500 AU*min), among these only one was female.

Fig.2: Rate of responsiveness to antiplatelet therapy.
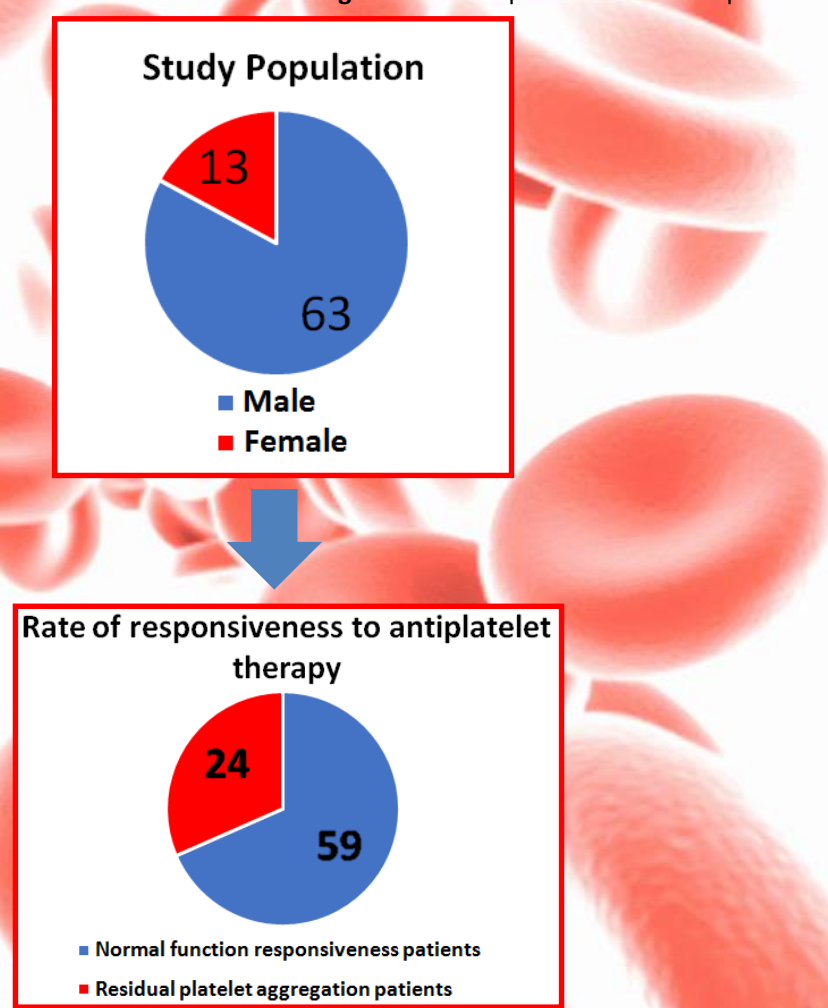

- Residual platelet aggregation patients

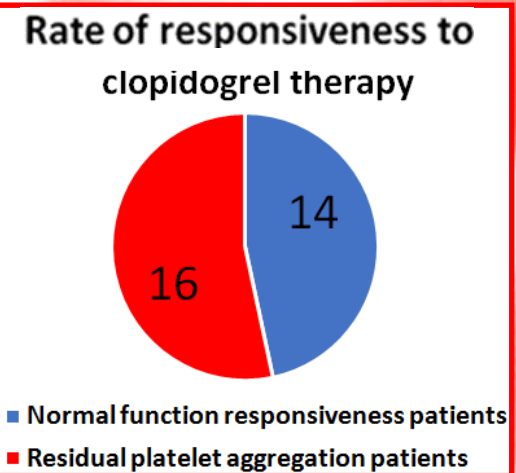

Rate of responsiveness to salicylates therapy

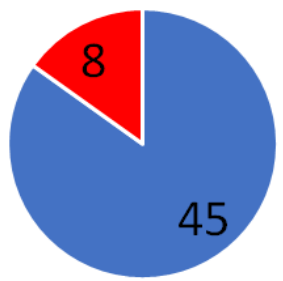

- Normal function responsiveness patients

- Residual platelet aggregation patients 


\section{FACTORS INFLUENCING THE ANTIPLATELET THERAPY IN PATIENTS REQUIRING VASCULAR SURGERY.}

Fig.3. Factors influencing clopidogrel therapy in study population.

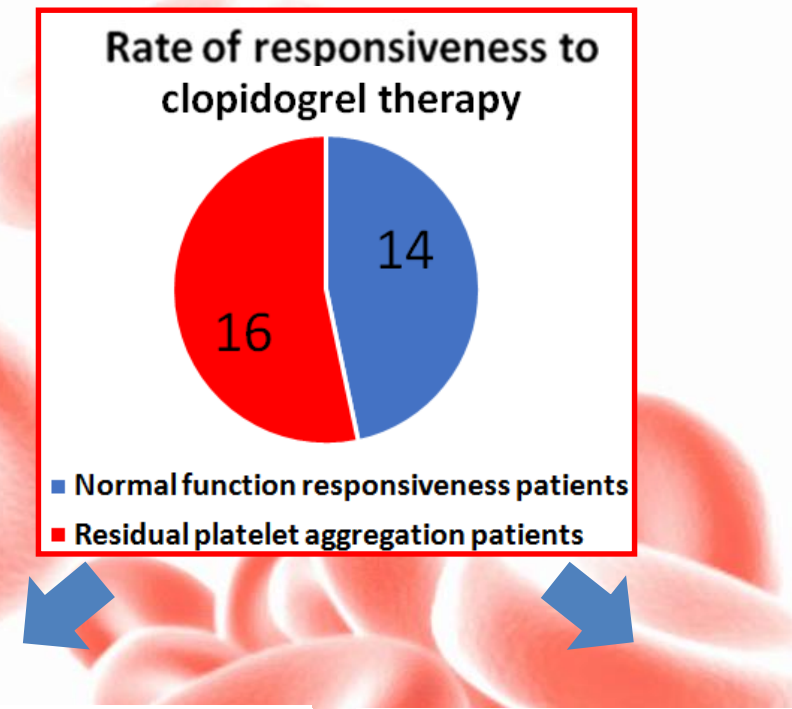

Genotypes in the patients treated with clopidogrel

PPI in patients on clopidogrel therapy

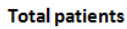

on clopidogrel

CYP2C19*1/*17

CYP2C19*1/*2

ABCB13435TT

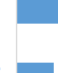

АВCB13435CT

Patients without PPI therapy

Patients on lansoprazole

Patients on esomeprazole

Patient on omeprazole

Total patients on clopidogrel

\section{Discussion and conclusions}

A relevant percentage (31.6\%) of the enrolled patients showed RPA. The variable response to AT is a problem occurring both for clopidogrel and salicylates, although it is more frequent for the first. It is important to monitor non-responders over time because they are at increased risk of ischemic events. Periodic control of platelet reactivity and the modification of the therapy should be planned case by case, taking into account all the factors recognized to influence the therapy.

\section{References}

1 Scott SA, Sangkuhl K Clinical Pharmacogenetics Implementation Consortium guidelines CYP2C19 genotype and clopidogrel therapy 2013 update. Clin Pharmacol Ther. 2013.

2 Galeazzi R, Olivieri F-Clustering of ABCB1 and CYP2C19 Genetic Variants Predicts Risk of Major Bleeding and Thrombotic Events in Elderly Patients with Acute Coronary Syndrome Receiving Dual Antiplatelet Therapy with Aspirin and Clopidogrel. Drugs Aging. 2018

3 Eusebi et al-2017-Journal of Gastroenterology and Hepatology; Ho PM, Maddox TM Risk of adverse outcomes associated with concomitant use of clopidogrel and proton pump inhibitors following acute coronary syndrome. JAMA. 2009 